\title{
LINE: Link Information Normalization Environment
}

\section{Umar Toseef}

\author{
Asanga \\ Udugama \\ Communication Networks Group \\ University of Bremen \\ 28359 Bremen, Germany \\ +49 (421) 218-[ 8665 | 8665 | 2277 | 8264] \\ [umr | adu |cg | fon]@comnets.uni-bremen.de
}

\author{
Frank \\ Pittmann \\ Nokia Siemens Networks \\ $\mathrm{GmbH} \&$ Co. KG \\ 13623 Berlin, Germany \\ +49 (30) 386-29387 \\ frank.pittmann@nsn.com
}

\begin{abstract}
Today, there exists a large variety of wireless networking technologies. However, the question is how to use all these technologies and the available services together to obtain the best possible advantage for the user and network operators. Currently research is going on over handover techniques that allow the user to seamlessly handover from one access to another. To optimize this process there is a need for a standard interface between link layer and upper layers as well as an information normalization mechanism that provides the link information independent of the access network and technology in use. This normalized link information then can be used to decide about switching from one access to another. This paper discusses a Link Information Normalized Environment (LINE) that can be used by upper layers for making decisions.
\end{abstract}

\section{Categories and Subject Descriptors}

C.2.1 [Computer-Communication Networks]: Network Architecture and Design - Network communications, Wireless communication

\section{C.2.3 [Computer-Communication Networks]: Network Operations - Network monitoring}

\section{General Terms}

Algorithms, Management, Performance, Design, Experimentation, Verification

\section{Keywords}

Link Normalization, Optimized Handovers, Mobility Management, Future 3GPP Radio

\section{INTRODUCTION}

This work is carried out within the project ScaleNet (Scalable, efficient and flexible Network) that is partly sponsored by the German Ministry for Education and Research within the framework 'Networks of Tomorrow'. One aspect of ScaleNet is the inter-working between 3GPP and non-3GPP networks

Permission to make digital or hard copies of all or part of this work for personal or classroom use is granted without fee provided that copies are not made or distributed for profit or commercial advantage and that copies bear this notice and the full citation on the first page. To copy otherwise, to republish, to post on servers or to redistribute to lists, requires prior specific permission and/or a fee. MOBILWARE 2008, February 13-15, Innsbruck, Austria

Copyright @ 2008 ICST 978-1-59593-984-5

DOI 10.4108/ICST.MOBILWARE2008.2889 considering mobility and other network services while efficiently supporting IMS applications, hence, aiming at inter-access system service delivery in cooperation with efficient, optimized mobility management between the accesses.

As a part of ScaleNet activities, this paper details the use of normalized link values to optimize handovers in mobility management protocols residing at layer 3 (MIP, FMIP, HMIP) and beyond (HIP, SIP). It considers all possible link layer technologies to provide normalized information to a decision engine, which utilizes this information comparing them against predefined values and deciding which network attachments are the most suitable as of a given moment. Most of the handover optimization techniques used in previous research pass individual L2 specific parameters to the upper layers.

Link Information Normalization Environment (LINE) is a component that obtains link information, does normalization and passes on to the upper layers. This normalized information can be used to make decisions such as optimized handovers by mobility management protocols, by application layer (e.g. IMS codec changes) and by policy based decision environments (e.g. POLIMAND [1], NetCAPE [6], Proton [7]). This paper details concept work of LINE, implementation architecture and the analysis of handover results between a legacy and a future 3GPP radio access taken in an experimental environment when using Mobile IPv6 as the mobility management protocol.

\section{RELATED WORK}

Link layer (L2) information can be used by upper layers and protocols to define hints and/or thresholds for handover decisions. Handover decisions based on this information are more reliable and easy to make. This section looks at some research in this area and their focus.

Policy Based Mobile IP Handover Decision (POLIMAND) defines a policy for different decisions for MIP handovers [1]. This policy, required for different available access networks, e.g. WLAN, GPRS, and UMTS defines when the current access network is no longer available or it does not fulfill the requirements or predefined quality parameters. In this case the policy is the source for the decision which access network has to be used by the mobile node. In order to implement policy decisions POLIMAND needs link quality information of the available networks from link layer. It uses Generic Link Layer (GLL) to obtain link information from the access systems.

The concept of a GLL was motivated by the requirements of wireless communication with a seamless and lossless cooperation between different access networks with the support of three 
additional functions [3]. They are, Cooperation with mobility management functions and protocols, Context transfer at handover and Lossless reconfiguration. Further, the GLL defines three different interfaces of the physical layer interface, the network layer interface and the control interface.

Although GLL meets the requirements of POLIMAND to access link information in a generic way, the GLL also provides a host of additional information and functionality that is not of much use for POLIMAND. Since POLIMAND is interested only in the link parameter information of the available access links, the rest of the information is meaningless in the context of POLIMAND. Moreover the goal of GLL is to provide a generic functionality to all of the upper layers and protocols which makes GLL a very complex and unwieldy entity. This implies that including the whole GLL architecture in POLIMAND does not seem appropriate. What is required is the part of GLL that can provide us with necessary information of link parameters in a generic way. This basically is the task of the LINE. LINE provides normalized L2 information to a policy engine like POLIMAND or NetCAPE.

Another similar concept is the IEEE 802.21 [2]. This upcoming standard attempts to provide optimizations on handovers between IEEE 802.11, 802.21 and 3GPP interfaces. It is a very heavy protocol that requires changes to MAC protocols, require interactions with network components to make decisions and consisting of simple non-numerical normalization mechanism (i.e. link up, link down, etc.). Further, it is also in control of the network layer to build the IP layer. LINE on this other hand, is a simple mechanism that simply provides information to the upper layers to make the handover decisions as the upper layers have much knowledge about the overall situation (e.g. application requirements).

\section{LINE ARCHITECTURE}

\subsection{LINE Concept}

LINE, in a conceptual level, encompasses all possible link layer technologies to provide normalized information to a policy engine, called NetCAPE (Networking Context Aware Policy Environment) to use in the determination of the handovers (Figure 1). These handovers are realized using any mobility management protocol.

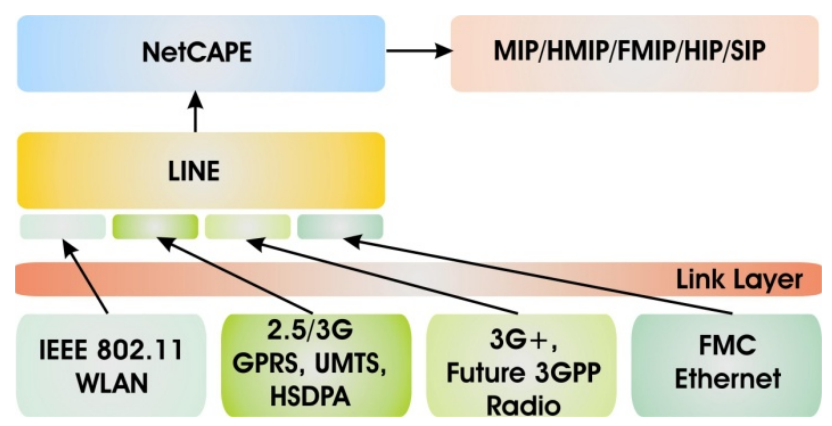

Figure 1. LINE architecture

The same parameters for different link technologies have different range of possible values. For example, RSSI in IEEE 802.11 has a range of values from 1 to 134, while in GSM it ranges from 1 to 31. Moreover there are also technology specific thresholds for weak, good and excellent link quality measures. The solution to these problems is to perform a normalization of parameters and describing them on a uniform scale. This uniform scale should be a generic link quality indicator that indicates the link quality independent of the access technology. We have defined a scale with the range of values from 0 to 100 and divided it into regions that describe different levels of link quality, as shown in Table 1.

This uniform scale has four levels of link quality indicators that are used by NetCAPE for further decision making. There are three different kinds of possible normalization mechanisms that were identified at a concept level.

Option I: An individual and independent normalization of L2 information is performed in this option by mapping it to this scale utilizing a linear mapping mechanism. An example is shown in Figure 2 showing the mapping that was obtained for a GPRS network interface. LINE is provided with a data base that contains the $\mathrm{L} 2$ values (BER in our example) related to the different levels obtained through a calibration process. This process identifies the different regions of quality of the link parameter. LINE obtains the L2 values from the link layer and does a mapping in a uniform manner considering the regions identified in a calibration process. This mapped value will represent the normalized link quality for that L2 parameter. The calibration process is where each network interface is used continuously, changing to different throughput regions (through movement or signal attenuation) and identifying the link quality for different throughput regions.

This option, although simple to implement and process, gives only a rough estimation of link quality. Since the normalization is based on the identified regions (using calibration process) of the individual parameters, the link quality of different technologies cannot be compared. In other words the link quality obtained this way is not a generic link quality indicator.

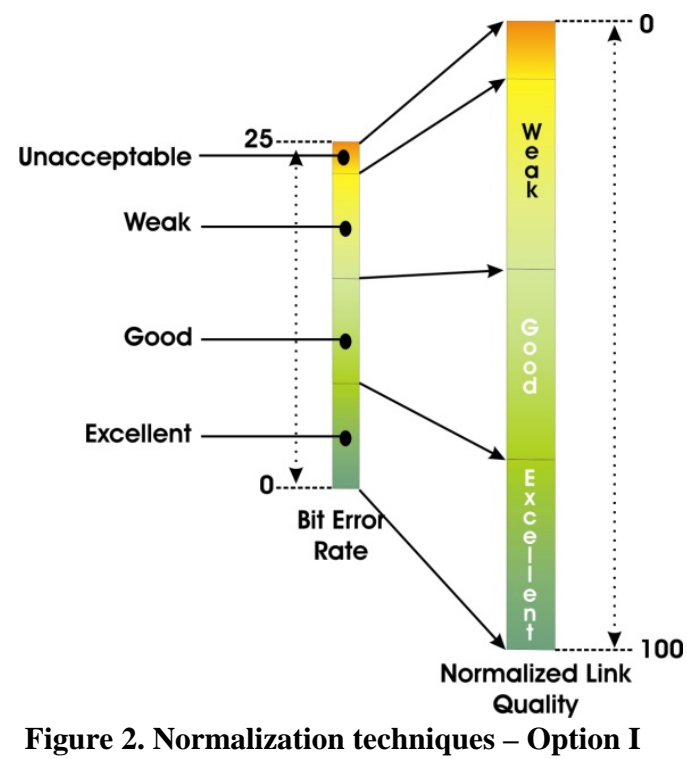

A question can arise with this option as to how a decision is made to identify the unacceptable, weak, good and excellent regions during the calibration process. To decide for unacceptable region is simple as it is the link quality in which no data services are available. For weak, good and excellent regions we can either decide for different percentage of maximum throughput or according to different modes of operation of the physical layer that are selected by hardware depending on link quality (such as 
WLAN PHY modes).

Option II: This option adopts the normalization of one single common L2 parameter (e.g., SNR). If a link does not provide this parameter, available link parameters such as BER have to be mapped or translated to the common value. Option I, suggests the normalization of the available L2 parameters individually. However in order to make comparison of link quality of different access technologies, it is appropriate to do normalization of one single common L2 parameter from all technologies. For this purpose one L2 parameter can be selected and if the required L2 parameter is not available, then the available L2 parameters can be translated to that selected L2 parameter (Figure 3). This L2 parameter can then be mapped to the uniform scale.

Considering an example to further clarify; assuming that SNR is selected as a standard L2 parameter that will represent the link quality for all access technologies. Then, for those interfaces that only provide RSSI and BER values instead of SNR, a translation will be made from RSSI and BER to SNR value. Hence it will be more logical to make comparison of these SNR values from different technologies to compare the link quality of these access technologies.

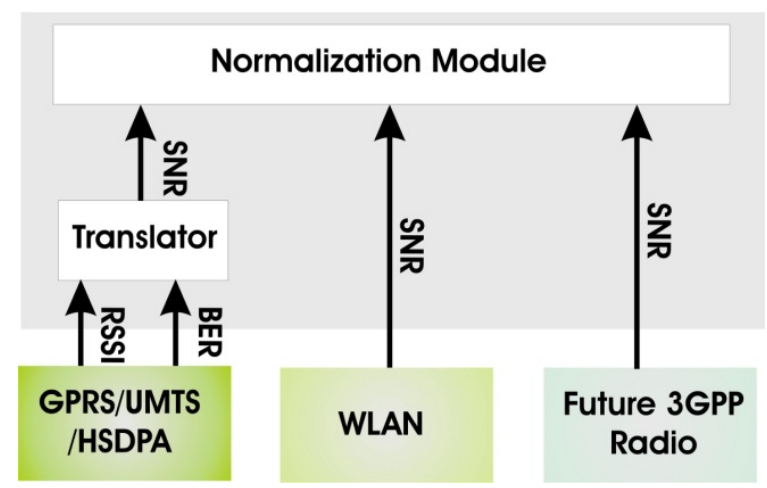

Figure 3. Normalization techniques - Option II

This option is better than option I in two ways. Firstly, we are comparing the identical L2 parameters. Secondly, in some cases we are using more than one L2 parameters where single L2 parameter cannot precisely reflect the link quality.

However there might be problems in the translation of L2 parameters. That is, how to translate different L2 parameters into other L2 parameters. Curves or empirical lookup tables specific to access technologies can be created by simulations or using actual traces for this translation.

Another problem is the significance of the parameters related to physical layer technology. For example, the SNR value that must be present to provide a satisfactory data service for UMTS will be different than that for WLAN because of different physical layer and link layer technologies. Due to this fact it is not possible to compare SNR values of different access technologies in order to compare the link quality of these access technologies.

Option III: The previous explanations shows that neither option I nor option II provide a comparable link quality metric. The reason for this is due to the significance that each access technology places on different L2 parameters. This results in not having a level playing field when comparing these L2 parameters. In order to build a generic link quality metric, it is better to go one layer up on the protocol stack and select some L3 parameter that is completely independent of the access technology. For example this L3 parameter can be the throughput at network layer. As the throughput depends obviously on link quality so it will give the perfect estimation of link quality. Since it is an L3 parameter, it will not matter what access technology is being used at the link layer or what is the current mode of operation of the device.

The problem however, is how to determine the current throughput of a given interface. There are two possible ways to determine the throughput.

One way is to send test data through the interface to determine the current achievable throughput. The advantage of this method is that there is no need to get L2 parameters which is sometime problematic (as explained earlier). However, the disadvantage of this method is that the link has to be flooded with the test data. The situation will be even worse if the access technology cannot support high data rates and the throughput has to be estimated more frequently.

The other way to determine the current achievable throughput is to perform some estimation depending on the current status of L2 parameters. This means a translation from L2 parameters to L3 throughput. This estimation or translation can either be performed theoretically with the help of simulation results or through a calibration process where a mapping is established between L2 parameters and the corresponding L3 throughput. The limitation of the simulation oriented method is its theoretical orientation as the practical situations may be very different from the simulated one. Similarly, the negative point of performing calibrations is that each network interface will have different results due to implementation variations (such as different algorithms, proprietary mechanisms, etc) of different manufacturers. However this problem can be solved by utilizing a dynamic (i.e. real time) calibration process unlike the way it is done in Option 1. That is, the mapping database is updated regularly by monitoring the user data instead of generating data for the sole purpose of determining throughput.

\subsection{LINE Implementation}

The ScaleNet project, under its initial phase, has developed a version of LINE that utilizes the option I. This implementation normalizes link information obtained from WLAN, UMTS, HSDPA, GPRS and a future 3GPP prototype radio interface (in the following briefly referred to as 'future 3GPP radio') (Figure 4). Additionally, Ethernet interfaces are also considered as part of the Fixed Mobile Convergence (FMC) extensions to LINE.

Under this work, LINE provides a Normalized Link Quality (NLQ) to NetCAPE which utilizes this information to perform handovers. NetCAPE runs this information through a hysteretic mechanism to prevent ping-pong effects and presents this information to a policy engine that considers the NLQ together with other information to instruct Mobile IPv6 to make handovers.

LINE consists of a set of modules that performs different tasks and interact with a control module to pass information to NetCAPE.

- WLAN module monitors the IEEE 802.11 interfaces by reading link information from the Linux proc file system.

- UMTS/GPRS/HSDPA modules deal with the respective access technologies. They retrieve link information (RSSI, $\mathrm{BER}$, etc) by issuing AT commands to the respective modem. 
- $\quad$ Ethernet module monitors whether Ethernet link is up or not and whether Ethernet cable is plugged or unplugged.

- $\quad$ Future 3GPP radio interfacing module is a web services based interface to the prototype future 3GPP radio module to obtain the QoS values that describe the link quality.

- The normalization module translates and maps the obtained link information to the uniform scale. The database contains the threshold values obtained through the calibration process which is used for the translation and mapping.

- $\quad$ The main module manages all the above modules to execute in a given configuration with the responsibility of communicating with NetCAPE to obtain configuration information and to supply the NLQs created by the Normalization module. The interface between NetCAPE and LINE is realized using a UNIX socket, which is used to pass formatted strings containing information of each network interface.

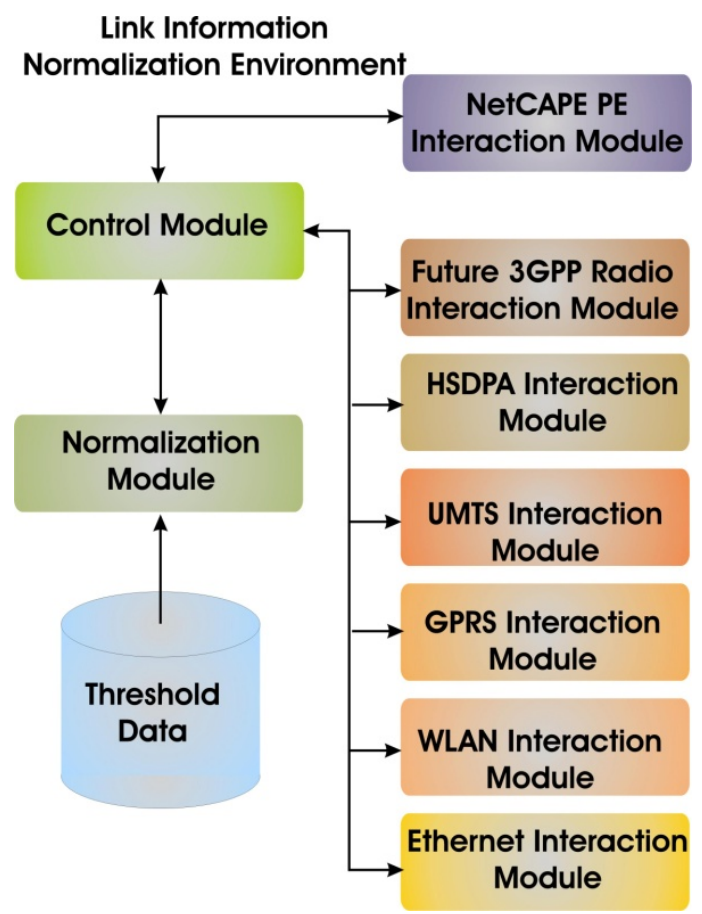

Figure 4. LINE implementation architecture

\section{PERFORMANCE TESTS AND THEIR ANALYSIS}

In order to evaluate the performance of MIP with and without LINE we performed two types of tests in a MIPv6 environment [4]. They are handover delay analysis and throughput analysis during handover.

These tests have been performed on a MN equipped with HSDPA and future 3GPP radio access technologies. We use a real NodeB and a network emulator emulating RNC, SGSN and GGSN for HSDPA and a future 3GPP prototype radio interface. MN has future 3GPP radio as its preferred access interface (quality and bandwidth is better compared to HSDPA) and is initially connected through it. Handover takes place as the signal quality of future 3GPP radio is changed using an attenuator. As the mobility management protocol, MIPv6 is used.

In the first experiment we ping a correspondent node with a 100 msec interval and a packet size of 64 bytes. Then, the link quality of future 3GPP radio is reduced so that handover to HSDPA takes place. During the handover process ping packets may be lost and total number of lost packets shows how appropriate was the handover decision and the timing. Table 1 shows the statistics of lost packets for 10 observations during handover from future 3GPP radio to HSDPA for two different router advertisement (RA) intervals.

It can be seen that LINE has greatly improved the performance of MIP handover by taking the handover decision in-time i.e. well before the link quality gets very bad. Moreover MIP without LINE also depends on RA interval while LINE makes MIP independent of RA interval. The reason for MIPs dependence on RA interval is that MIP waits for RA over the in-use interface and when it does not receive any RA in an expected period of time then it makes a handover to the other available interface. Shorter the RA interval more the link will be flooded with RA packets. Hence LINE not only helps in realizing a smooth and nearly lossless handover but also saves bandwidth required due to shortening of RA interval to improve MIP performance compared to without LINE.

Standard deviation of the 10 observations is also higher for MIP without LINE as compared to MIP with LINE. Standard deviation increases as RA interval increases for the same reason described above.

In the second experiment we monitor UDP and TCP throughput variations during the handover from future $3 \mathrm{GPP}$ radio to HSDPA. RA interval has been selected to be 18-24 sec.
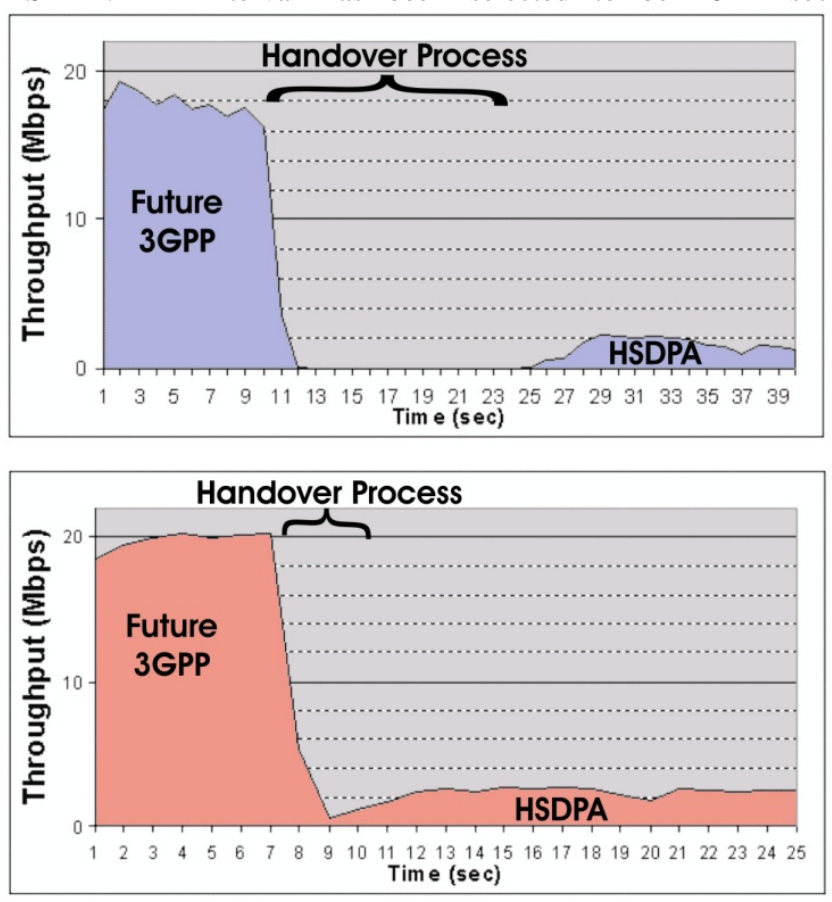

Figure 5. TCP throughput variations without LINE (top) and with LINE (bottom)

The Figure 5 shows the TCP throughput graphs obtained using IPerf [5]. MIP without LINE suffers from long period of zero 
throughput, while MIP with LINE significantly improves the handover process without any throughput reductions to zero. The 'Handover Process' period represents the total time during which MIP figures out that it is no more connected to the current interface and hence performs a handover to one of the available interfaces. Since MIP without LINE solely depends on RA receptions, it takes longer for MIP to figure out that the in-use link is now down and it should do a handover. But LINE by monitoring the link quality figures out in-time that the link will go down shortly and hence instructs MIP to perform a handover. So it is clear that longer the RA interval is (due to MIP without LINE), longer the 'Handover Process' period.
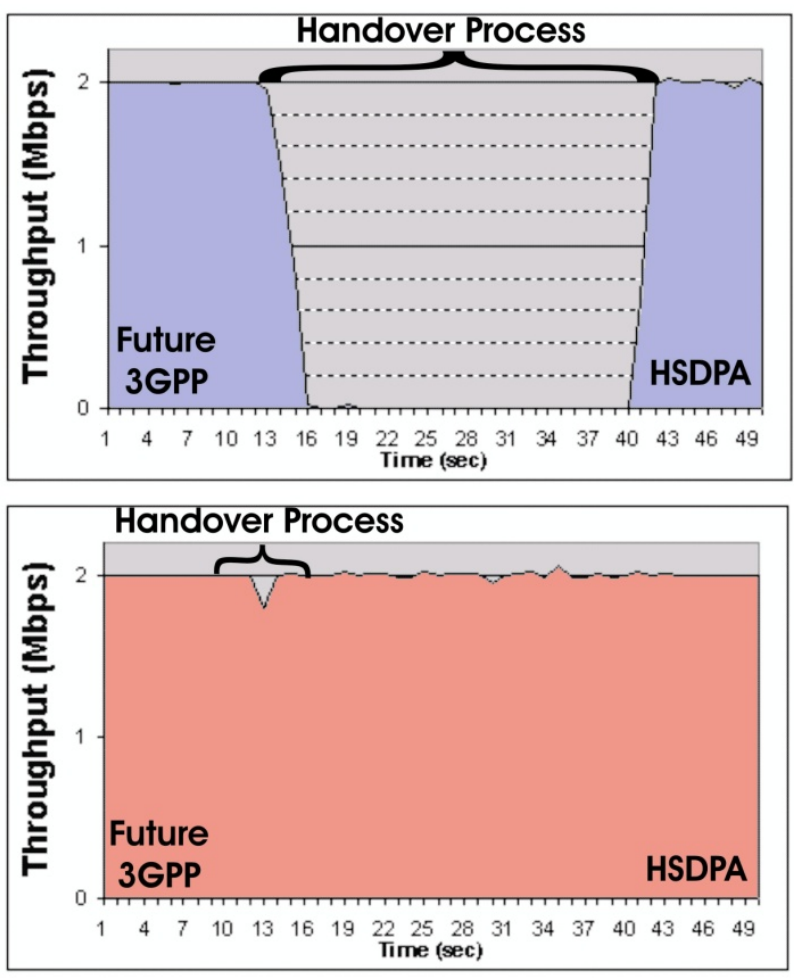

Figure 6. UDP throughput variations without LINE (top) and with LINE (bottom)

Figure 6 shows the UDP throughput measured using IPerf [5] limiting maximum throughput to $2 \mathrm{Mbps}$. Again it can be seen that MIP without LINE suffers from a long period of zero throughput before doing handover to HSDPA while throughput of MIP with LINE support is almost unaffected by the handover process.

\section{CONCLUSIONS}

LINE provides normalized L2 information to the above layers in a uniform scale. This information can be used by any upper layer protocol to make decisions. This paper discussed how the normalized L2 information given by LINE can be used by one of the mobility management protocols, i.e. MIPv6 to enhance the handover decisions. Experimental evaluation showed the improvement in performance during handovers between 3GPP (i.e. HSDPA) and future 3GPP radio interfaces (a prototype developed within ScaleNet project). Individual mapping of L2 information to a normalized value (i.e. option I) has been implemented in LINE for the first version of the ScaleNet demonstrator. This demonstrator with NetCAPE will be shown at $3 G$ world congress, held in December, 2006 in Hong Kong. IMS based video conferencing and video supervision (VSS) applications are used as user applications. Information provided by LINE (via NetCAPE) will be used by IMS applications to adapt the video/audio quality for the change of link qualities.

For further demonstrations within the ScaleNet project, LINE will be modified to have options 2 and 3 which are explained in section 3.1. LINE information will be given to the other protocols like SIP, FMIP to make decisions.

\section{REFERENCES}

[1] S. Aust, C. Pampu, and C. Görg. Proactive Handover Decision for Mobile IP based on Link Layer Information. First IFIP International Conference on Wireless and Optical Communications Networks (WOCN), June 2004.

[2] IEEE 802.21, http://www.ieee802.org/21/archived_docs/Documents/802_H andoff_CFIr1.pdf

[3] Sachs, A Generic Link Layer for Future Generation Wireless Networking, ICC, May 11-15, 2003, Anchorage, Alaska, USA

[4] RFC 3775: Mobility support in IPv6, June 2004

[5] Tirumala, A., et al., Iperf: The TCP/UDP Bandwidth Measurement Tool, avialable at http://dast.nlanr.net/Projects/Iperf.

[6] NetCAPE (Networking Context Aware Policy Environment), internal deliverable, ScaleNet Project

[7] P. Vidales, R. Chakravorty, and C. Policroniades. PROTON: A Policy-based Solution for Future 4G devices. Proceedings of the IEEE International Workshop on Policies for Distributed Systems and Networks, (IEEE POLICY 2004), June 2004. 